\title{
$p 53$ gene cloning and response to hypoxia in the plateau zokor, Myospalax baileyi
}

\author{
Zhi-fang An ${ }^{1,2,+}$, Kang Zhao ${ }^{3,+}$, Lin-na Wei ${ }^{4}$, Zhi-jie Wang ${ }^{4}$, Su-hua $\mathbf{L i}^{2}$, \\ Lian Wei ${ }^{4}$ and Deng-bang Wei ${ }^{1,4, *}$ \\ ${ }^{1}$ State Key Laboratory of Plateau Ecology and Agriculture, Qinghai University, 251 Ningda Road, \\ Xining, Qinghai 810016, China \\ ${ }^{2}$ Research Center for High Altitude Medicine, Qinghai University, 251 Ningda Road, Xining, \\ Qinghai 810016, China \\ ${ }^{3}$ College of Medical, Qinghai University, 251 Ningda Road, Xining, Qinghai 810016, China \\ ${ }^{4}$ College of Eco-Environmental Engineering, Qinghai University, 251 Ningda Road, Xining, \\ Qinghai 810016, China
}

Submitted: January 5, 2018. Final revision received: March 3, 2018. Accepted: April 2, 2018

\begin{abstract}
The plateau zokor (Myospalax baileyi) is a specialized subterranean rodent that lives on the QinghaiTibet Plateau. The species has evolved a series of strategies to adapt to its hypoxic environment and hypercapnia. p53 is a tumour suppressor gene that plays a crucial role in the cellular response to hypoxia by inducing cell cycle arrest, cell apoptosis, DNA damage repair and angiogenesis. To investigate the sequence characteristics of $p 53$ and the response to hypoxia in plateau zokor, we cloned the p53 coding DNA sequence, analysed it, and measured the expression level of $p 53$ at different altitudes in plateau zokor and rats. Our results show that the coding DNA sequence is $1179 \mathrm{bp}$, consisting of 392 amino acid residues. Compared to human $p 53$, the subterranean rodents have two mutation sites in common with the human hotspots in the DNA-binding domain. Compared to subterranean rodents, plateau zokor have a mutation at residue 309. In addition, subterranean rodents have two convergent sites at residues 78 and 84 . The expression levels of $p 53$ in plateau zokor tissues increase significantly from $2260 \mathrm{~m}$ to $3300 \mathrm{~m}$, but there was no significant difference in rats at those altitudes. Our results suggest that subterranean rodents have two mutation sites in common with the human hotspots in the DNA-binding domain, the mutation of Gly309Asp is a unique mutation site of plateau zokor $p 53$, and there are two convergent sites enhancing subterranean rodent adaptation to hypoxic conditions. In addition, $p 53$ is sensitive to the oxygen concentration in plateau zokor, and hypoxia upregulates the levels of $p 53$. Generally, plateau zokor use this strategy to adapt to a hypoxic environment.
\end{abstract}

+) These authors contributed equally to this work.

*) Corresponding author; e-mail: weidengbang@163.com 


\section{Keywords}

Asiatic burrowing rodent; hypoxic; p53; rodent; TP53; tumor protein; tumour suppressor

\section{Introduction}

p53, a tumour suppressor gene, plays a major role in the cellular response to DNA damage and hypoxia (Resnick \& Inga, 2003; Ashur-Fabian et al., 2004; Resnick et al., 2005; Menendez et al., 2006). It is also one of the most frequently mutated proteins in cancers (Hernandez et al., 2003; Klein, 2004). Human p53 has 393 residues, and it contains a transcriptional activation domain (TAD), a sequencespecific DNA-binding domain (DBD), a tetramerization domain (TD), a C terminus (CT), and a proline-rich domain (PRD) (Bargonetti et al., 1993; Cho et al., 1994; Thut et al., 1995; Walker \& Levine, 1996). The DNA-binding domain is the core domain of $p 53$, and a majority of mutations occur in this region (Pavletich et al., 1993; Zhao et al., 2001). The $p 53$ gene has two types, wild and mutant. The wildtype $p 53$-encoded protein suppresses tumourigenesis by regulating cell cycle arrest, cell apoptosis (Vousden \& Lu, 2002; Klein, 2004), DNA damage repair and angiogenesis (Graeber et al., 1996; Kinzler \& Vogelstein, 1996). This protein has been called the "guardian of the genome" and a "gatekeeper for growth and division" (El-Deiry, 1998). However, the mutant $p 53$-encoded protein loses its anti-tumour function and can also promote tumourigenesis, including in several human cancers (Vousden \& Lu, 2002; Menendez et al., 2006). The biological effects of p53 can be explained by its ability to activate the expression of a number of target genes. These target genes can mediate cell cycle arrest, as with $p 21,14-3-3-\delta$, and Reprimo, induce cell apoptosis, as with Apaf-1, Fas, and Bax, or inhibit angiogenesis and metastasis, as with PAI, TSP1, and KAI (El-Deiry, 1998).

Subterranean rodents are small mammals that spend their entire lives underground in sealed burrows. Severe hypoxia and hypercapnia is common in burrows (Fan \& Shi, 1982; Nevo, 1999, 2011; Nevo et al., 2001; Shams et al., 2005). In evolution, subterranean rodents have over the long term developed a series of phenotypic, physiological and genomic strategies to adapt to the harsh environment. Firstly, the eyes of subterranean rodents are degenerated, but they display photosensitivity in their photoperiodic response. These rodents have also developed skeletal muscle that is conducive to digging burrows (Pevet et al., 1984; Nevo, 1999; Fang et al., 2014). Secondly, in these animals the surface area of the pulmonary alveoli and the alveolar capillary volume are enlarged. This change benefits the lungs by increasing the capacity for oxygen uptake (Widmer et al., 1997; Wang et al., 2008a). Thirdly, the increased erythrocyte count and haemoglobin and myoglobin content allows for efficient oxygen transport (Arieli et al., 1986; Wei et al., 2001a, b, 2006). Fourthly, they have reduced the distance for oxygen diffusion to the mitochondria, which ensures efficient oxygen delivery by the structural adaption of tissues (Arieli \& Ar, 1981; Edoute et al., 1988). A series of genes have also been shown to be adapted at the molecular level to the underground habitat. This 
modification includes a higher expression level for genes like hypoxia-inducible factor $1 \alpha(H I F-1 \alpha)$, vascular endothelial growth factor $(V E G F)$, and erythropoietin (EPO) (Shams et al., 2004, 2005; Ke \& Costa, 2006; Zheng et al., 2011; Wang et al., 2012), and the substitution of amino acids in haemoglobin, haptoglobin and myoglobin (Gurnett et al., 1984; Kleinschmidt et al., 1984, 1985; Ben-Shlomo \& Maeda, 1989). In addition, subterranean rodents display exceptional longevity. Heterocephalus glaber and Spalax judaei have maximum lifespans exceeding 30 years (Buffenstein \& Jarvis, 2002; Buffenstein, 2008; Kim et al., 2011; Tian et al., 2013) and 21 years (Edrey et al., 2012), respectively. In addition to longevity, reporter assays have shown a striking resistance to cancer. Multi-year observations of large samples did not detect a single incidence of cancer (Buffenstein \& Jarvis, 2002; Buffenstein, 2008; Delaney et al., 2013).

Previous $p 53$ studies revealed that an arginine (R) was substituted by lysine (K) in residue 172 (Arg174 in human) in Spalax. This substitution is similar to certain mutations found in human tumours; therefore, it implies that Spalax undergoes similar adaptations to hypoxia to those undergone by $p 53$ in human cancers. The mutation reduces the transcription of apoptosis genes, such as Apaf-1, Puma, Pten and Noxa, and enhances the cell cycle arrest and p53 stabilization/homeostasis genes $M d m 2, p 21$ and $C y c G$ (Ashur-Fabian et al., 2004; Avivi et al., 2007). Genome and transcriptome analysis of the $p 53$ signal pathway has shown that eight p53 target genes are differentially regulated by hypoxia in Spalax and rat (Fang et al., 2014).

The plateau zokor, Myospalax baileyi, is a specialized subterranean rodent living on the Qinghai-Tibet Plateau. This species inhabits sealed burrows at an altitude of 2800 to $4200 \mathrm{~m}$ (Wang et al., 1979; Fan \& Shi, 1982). Like other subterranean rodents, the plateau zokor has strategies to adapt to hypoxic and hypercapnic underground environments. In $M$. baileyi, the $p 53$ expression level was significantly decreased in the liver when mimicking oxygen levels at an altitude of $7000 \mathrm{~m}(8.0 \%$ $\mathrm{O}_{2}$ ) for $8 \mathrm{~h}$ compared to an altitude of $3352 \mathrm{~m}$ (Wang et al., 2013; Zhao et al., 2013). Comparison of $p 53$ sequence data and functional studies found that a mutation in codon 104 from serine $(\mathrm{S})$ to asparagine $(\mathrm{N})$ activated the apoptosis-related genes IGFBP3, Apaf-1 and Bax, but had no significant influence on cell cycle arrest genes, including $p 21$ and $M d m 2$ (Zhao et al., 2013).

To date, the sequence characteristics of $p 53$ and the response to hypoxia in the plateau zokor are not understood well. In this study, we clone the coding DNA sequence (CDS) of $p 53$, analyse and characterize $p 53$ in other rodents, and measure $p 53$ 's expression differences between high and low altitudes in plateau zokor tissues using quantitative real-time PCR. 


\section{Materials and methods}

\section{Animals and sample collection}

Plateau zokors were live-trapped in the Zongjiagou region in Huangyuan country, Qinghai Province, China. They were divided into two groups: (1) high altitude (3300 m group), which were zokors collected from the Zongjiagou region in Huangyuan country at an altitude of $3300 \mathrm{~m}$ with an oxygen content of $193.4 \mathrm{~g} / \mathrm{m}^{3}$; (2) low altitude $(2260 \mathrm{~m})$, which were zokors collected from Zongjiagou in the Huangyuan region and raised in Xining City, Qinghai Province, China at an altitude of $2260 \mathrm{~m}$ for eight days with an oxygen content of $226.7 \mathrm{~g} / \mathrm{m}^{3}$. Sprague-Dawley (SD) rats were bought from Lanzhou, Gansu Province, China. Rats were divided into two groups: (1) high altitude (3300 m Group), raised in Zongjiagou region in Huangyuan country for eight days; (2) low altitude $(2260 \mathrm{~m})$, raised in Xining City at an altitude of $2260 \mathrm{~m}$ for eight days. The sample size was 8 for each of the above groups. All animals were anaesthetized with sodium pentobarbital (5\%) and sacrificed using cervical dislocation immediately before dissection. Liver, lung, stomach, intestine and skeletal muscles were rapidly removed and quickly frozen in liquid nitrogen. All procedures involved in the handling and care of animals were in accordance with the China Practice for the Care and Use of Laboratory Animals and approved by the China Zoological Society (permit number: GB 14923-2010).

\section{Cloning of the p53 CDS sequence of plateau zokor}

The plateau zokor p53 CDS was cloned from mRNA extracted from the liver. Primers were designed according to the Homo sapiens (DQ263704), Rattus norvegicus (AH002222), Mus musculus (AB020317), Cricetulus griseus (U50395), Eospalax cansus (JX998172), H. glaber (KM486789) and Microtus oeconomus (JX998171) p53 cDNA sequences. Total RNA was isolated using TRIzol reagent (Invitrogen Corp., Carlsbad, CA, USA). The RNA concentration and purity were assessed using UV spectrophotometry $(1.8<$ A260/A280 < 2.0). RNA integrity was checked using electrophoresis. A reverse transcription reaction was performed starting from $3.8 \mu \mathrm{g}$ of total RNA and using the First Strand cDNA Synthesis kit (TIANGEN, China). The PCR primers were designed as follows: forward, 5'-ATGGAGGAACCCCAGTCAG-3' and reverse, $5^{\prime}$-CAGGCGGAGGTGTGGAGTCG-3', with an amplicon length of $519 \mathrm{bp}$; forward, 5'-GCACGCAGTTCTGGAGGT-3' and reverse, 5'-AATTTCTCGAAGT CGGTGGT-3', with an amplicon length of $682 \mathrm{bp}$. A PCR reaction system of $25 \mu \mathrm{l}$ was used which contained $1 \mu \mathrm{l}$ template, $2.5 \mu \mathrm{l} 10 \times$ Taq buffer $\left(\mathrm{Mg}^{2+}\right.$-free), $2 \mu \mathrm{l}$ $\mathrm{MgCl}_{2}(25 \mathrm{mM}), 2 \mu \mathrm{l}$ dNTP mixture $(2.5 \mathrm{mM} /$ each $), 1 \mu \mathrm{l}$ forward and $1 \mu 1$ reverse primers $(10 \mu \mathrm{M}), 0.25 \mu \mathrm{l}$ Taq polymerase $(5 \mathrm{U} / \mu \mathrm{l})$ (Takara Bio Inc., Otsu, Shiga, Japan) and $15.25 \mu \mathrm{l}$ doubly distilled $\mathrm{H}_{2} \mathrm{O}$. PCR included denaturation at $95^{\circ} \mathrm{C}$ for $3 \mathrm{~min}$, followed by 35 cycles of $95^{\circ} \mathrm{C}$ for $30 \mathrm{~s}, 52^{\circ} \mathrm{C}\left(51^{\circ} \mathrm{C}\right)$ for $30 \mathrm{~s}$ and $72^{\circ} \mathrm{C}$ for $45 \mathrm{~s}$, and finishing with chain extension at $72^{\circ} \mathrm{C}$ for $5 \mathrm{~min}$. The amplified product was separated on a $1.5 \%$ agarose gel. The target product was recycled and purified 
with the TIANgen Midi Purification Kit (Tiangen Biotech (Beijing) Co., Beijing, China), cloned into the pMD 19-T vector (Takara Bio) and transformed into DH5 $\alpha$ competent cells. Positive clones were sequenced. The GenBank accession number is KX823344.

\section{Multiple alignment and phylogenetic tree construction}

The $p 53$ sequences were obtained from GenBank (table S1). A phylogenetic tree of p53 was constructed with ClustalW2 (http://www.ebi.ac.uk/Tools/msa/clustalw2) and MEGA 7.0 software (Kumar et al., 2016) using the neighbour-joining method (Saitou \& Nei, 1987). Bootstrap re-sampling was applied to assess support for individual nodes using 1000 replicates. An amino acid sequence alignment of $p 53$ proteins from H. sapiens, M. baileyi, E. cansus, S. judaei, H. glaber and R. norvegicus was generated using Multalin software (http://multalin.toulouse.inra.fr/multalin/) (Corpet, 1988).

HIF-1 $\alpha$ sequences of $H$. sapiens (NM_001530.3), M. baileyi (DQ229099.1), and $R$. norvegicus (NM_024359.1) were obtained from GenBank. An amino acid sequence alignment of the HIF-l $\alpha$ oxygen-dependent degradation (ODD) domain from $H$. sapiens, $M$. baileyi and $R$. norvegicus was generated using Multalin software (http://multalin.toulouse.inra.fr/multalin/) (Corpet, 1988).

\section{Selection pressure analysis}

Sequence alignments were obtained using ClustalX1.81 (Jeanmougin et al., 1998), and format conversion was performed with MEGA 7.0 software (Kumar et al., 2016). The maximum-likelihood method was used to estimate the positive sites using branch-site models in codeml from the PAML package based on the mammalian species tree. The significance of the likelihood ratio test (LRT) statistic was determined using a $\chi^{2}$ distribution, and the positively selected sites were identified using Bayes Empirical Bayes (BEB) analysis (Zhang et al., 2005; Yang, 2007).

\section{Convergent evolution analysis}

Ten mammalian species were used to infer the convergent sites. We constructed ancestral p53 amino acid sequences using the Ancestors programme in the MEGA 7.0 software (Tamura et al., 2011). Ancestral inferences appeared reliable because mean posterior probabilities for the entire protein exceeded $99 \%$ for all nodes. We attempted to identify convergent changes by comparing ancestral and extant $p 53$ protein sequences. Next, we calculated the probability that the observed convergent sites exceeded the expectation due to random chance using the Jones-TaylorThornton (JTT) and Poisson models (Zhang \& Kumar, 1997).

\section{RNA extraction and quantification of $\mathrm{p} 53 \mathrm{mRNA}$ using qRT-PCR}

Total RNA was isolated from the liver, lung, stomach, intestine and skeletal muscle using TRIzol reagent (Invitrogen Corp.). The RNA concentration and purity were 
assessed using UV spectrophotometry $(1.8<$ A260/A280 < 2.0). RNA integrity was checked using electrophoresis. A reverse transcription reaction was performed starting with $3.8 \mu \mathrm{g}$ of total RNA and the First Strand cDNA Synthesis kit (Tiangen).

Quantitative real-time RT-PCR was performed using the SYBRPremix Ex

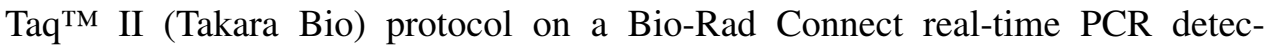
tion system (Bio-Rad Laboratories, Hercules, CA, USA) to quantify the expression level of $p 53$. qRT-PCR was performed at $95^{\circ} \mathrm{C}$ for 3 min followed by 40 cycles of $95^{\circ} \mathrm{C}$ for $30 \mathrm{~s}$ and $60^{\circ} \mathrm{C}$ for $30 \mathrm{~s}$. $\beta$-actin was used as an internal control. The PCR primers for $p 53$ and $\beta$-actin were designed as follows: zokor-p53: forward, 5'-GCCCAAGAAGAAGCCACTAC-3' and reverse, $5^{\prime}$-CCTGCTCTCTCCTGACTCCT-3', with an amplicon length of $142 \mathrm{bp}$; rat-p53: forward, 5'-CGACTATACCACTATCCACTACA-3' and reverse, 5'-GTCTTCCA GCGTGATGATG-3', with an amplicon length of $97 \mathrm{bp} ; \beta$-actin: forward, $5^{\prime}$-TCA CCAACTGGGACGATATG-3' and reverse, $5^{\prime}$-GTTGGCCTTAGGGTTCAGAG$3^{\prime}$, with an amplicon length of $119 \mathrm{bp}$. The relative expression level of p53 mRNA was computed based on the internal control gene using the $2^{-\Delta \Delta C T}$ method (Livak \& Schmittgen, 2001).

\section{Statistical analysis}

Statistical analyses were performed using SAS 8.2 software. The expression levels of $p 53$ between high and low altitudes were compared using Student's $t$-test. A value of $P<0.05$ was considered to be statistically significant.

\section{Results}

Comparison and phylogenetic tree of Myospalax baileyi p53 sequences

The p53 CDS was cloned and sequenced from M. baileyi. The complete coding sequence was $1179 \mathrm{bp}$, and the deduced protein consisted of 392 amino acid residues. Alignment by ClustalW2 found that the M. baileyi $p 53$ protein is identical to $98.98 \%, 95.41 \%, 87.02 \%, 82.99 \%$ with E. cansus, S. judaei, H. glaber and $R$. norvegicus $p 53$ proteins, respectively. The phylogenetic tree demonstrates that plateau zokor had the highest homology with E. cansus, followed by $S$. judaei (fig. 1).

Multiple sequence alignment analysis using Multalin software showed that compared with human $p 53$, four amino acid sequences of subterranean rodents $(M$. baileyi, E. cansus, S. judaei, H. glaber) had seven mutation sites in common within the DNA-binding domain: Met131Leu (M131L, 133 in human - numbering by actual position; numbering of the amino acids including gaps was used for the figure), Gln163Lys (Q163K, 165 in human), Val201Ala (V201A, 203 in human), Gln246Arg (Q246R, 248 in human), Leu265Arg (L265R, 267 in human), His271 Arg (H273R, 273 in human), and Leu287Phe (L287F, 289 in human) (fig. 2). 


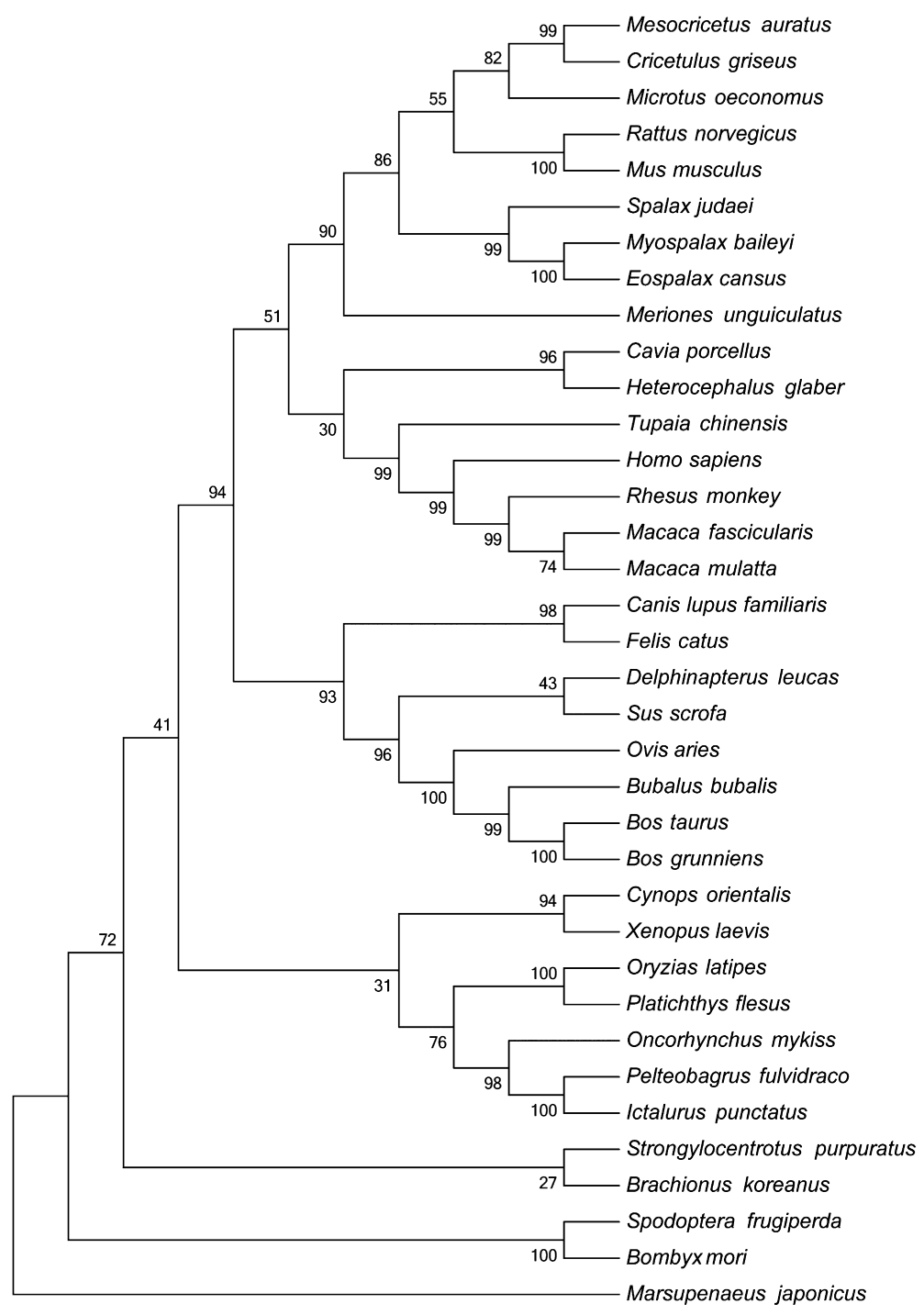

Figure 1. Phylogenetic tree based on the amino acid sequence of $p 53$ from Myospalax baileyi together with 36 other species. The $p 53$ sequences of the other species are from GenBank. Neighbour-joining method with 1000 bootstrap replicates was used. Bootstrap values for the internal nodes are given.

Compared with E. cansus p53, M. baileyi has two mutation sites within the DNA-binding domain: Ser104Asn, and Ala121Thr; compared with $S$. judaei p53, M. baileyi has three mutation sites within the DNA-binding domain: Ser104Asn, Pro127Cys, and Lys172Arg. By comparison with H. glaber $p 53$, there are fifteen mutation sites within the DNA-binding domain of $M$. baileyi: His98Gln, His108Arg, Gln113His, Val127Cys, Val143Leu, Glu146Asp, Pro148Thr, Arg207Lys, Thr208His, Asp219Glu, Leu220Pro, His288Arg, 


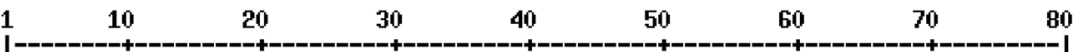

H.sapiens MEEPQSDPSHEPPLSQETFSDLHKLLPEHWYLS-PL--PSQHHDDLHLSPDDIEQHFTEDPGPDEAPRHPEFIPRYAPAP

H.sapilens

E.cansus

5. judaei

H.glaber

R, norvegicus

Consensus

$H_{\text {s sapiens }}$

H,baileyi

E.cansus

S. judaei

H. glaber

$R_{\text {+ norvegicus }}$

Consensus

H. sapiens

H,baileyi

E.cansus

S. judaei

H.glaber

$R_{+}$norvegicus

Consensus

H.sapiens

H.baileyi

E+cansus

S. judaei

H.glaber

R. norvegicus

Consensus

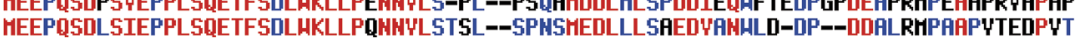
MEEPQSDLSIEPPLSQETFSDLAKLLPQHNWLSTSL--SPHSHEDLLLSAEDWANHLD-DP--DDALRMPAIAPUTEDPTT HEEQQSDLSIEPPLSQETFSDLAKLLPQHNYLSTPL--SPHSHEDLLLSPEDYAHHLD-DP--DERLQYPAHAITGDPUT HEEPQSDLSIEPPLSQETF SDLAKLLPEHHYLSSL--S-SPHDDLLLSPEDYYNHLGGHP--DEDYQYSAIFPYPEPPTP HEDSQSDHSIELPLSOETFSCLAKLLPPDDILPTTATGSPNSHEDLFL-PQDYHELLEGP---EEALQV-SFPARQEPGT

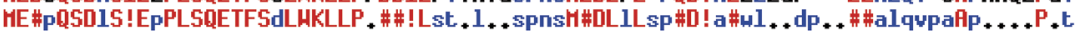

$\begin{array}{lllllllll}81 & 90 & 100 & 110 & 120 & 130 & 140 & 150 & 160\end{array}$

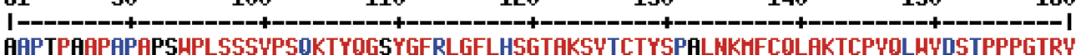
EASAPUAPPPATPHPLSSSUPSOKTYQGHYGFRLGFLHSIGTAKSWTCTYSPCLHKLFCQLAKTCPWOLHYDSTPPPGTRU EASAPWHPPPATPAPLSSSYPSQKTYQGSYGRLGFLHSGTAKSWACTYSPCLHKLFCQLAKTCPWQLHYDSTPPPGTRY

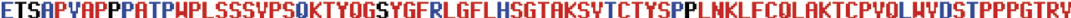
WAPAPAHPAPATSHPLSSSWPSHKTYQGHYGFHLGFLQSGTAKSWTCTYSPYLNKLFCQLAKTCPWQWHYESPPPPGTRW EAPAPWAPASATPHPLSSSWPSQKTYQGHYGFHLGFLQSGTAKSWMCTYSISLHKLFCQLAKTCPUQLHYTSTPPPGTRY

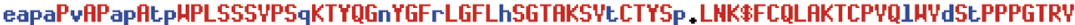

$\begin{array}{lllllllll}161 & 170 & 180 & 190 & 200 & 210 & 220 & 230 & 240\end{array}$

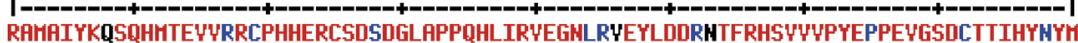
RAHAIYKKSDHHTEVYRRCPHHERCSDSDGLAPPQHLIRVEGHLRAEYLDDKHTFRHSYYUPYEPPEVGSDCTTIHYWYH RAHAIYKKSQHHTEYYRRCPHHERCSDSDGLAPPQHLIRVEGNLRAEYLDDKHTFRHSYYYPYEPPEYGSDICTTIHYNYH RAHAIYKKSQHHTEWYKRCPHHERCSDSDGLAPPQHLIRYEGHLRAEYLDDKHTFRHSWYPYYPPEVGSDCTTIHYWYH RAHATYKKSOHHTEUVRCPHHERCSDSDGLAPPOHLIRVEGNLRAEYLDDRTTFRHSYYUPYDLPEVGSDICTTIHYWYH RAHAIYKKSQHHTEYYRRHPHHERCSDGDGLAPPQHLIRYEGNPYAEYLDDRQTFRHSYYYPYEPPEYGSDYTTIHYKY'I

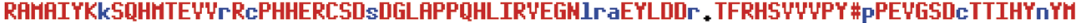

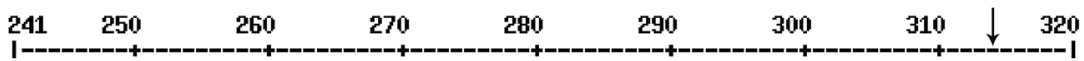

CHSSCHGGHNQRPILTIITLEDSSGNLLGLNSFEYHYCACPGRDRRTEEENLRKKGEPHHELPPGSTKRHLPNATSSSPQ

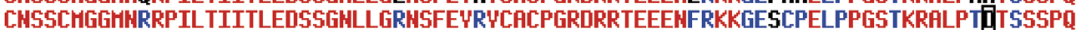

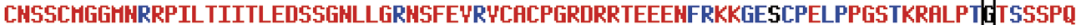
CHSSCHGGHNRRPILTIITLEDSSGHLLGRHSFEURWCACPGRDRRTEEEHFRKKGELCPELPPGSTKRHLPTGTSSSPQ

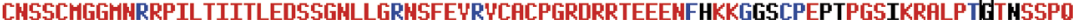
CHSSCMGGHINRPILTIITLEDSSGNLLGRDSFEVRVCACPGRDRRTEEENFRKKEEHCPELPPGSAKRHLPTSTSSSPQ

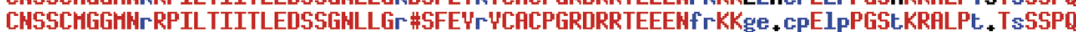

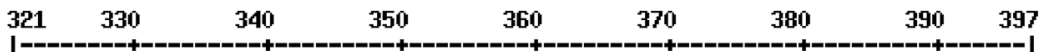

H,sapiens PKKKPL-DGEYFTLQIRGRERFEMFRELHEALELKDHQHGKEPGGSRHHSSHLKSKKGQSTSRHKKLHFKTEGPDSD

H,baileyi PKKKPLLDGEYFTLKIRGRERFEHFRELHEHLELKDHQHEKESGESRHHSSYLKSKKGQSTSRHKKLHIKREGPDSD

E,cansus PKKKPULDGEYFTLKTRGiRERFEHFRELHEALFLKDADAEKESGESRAHSSYLKSKKGOSTSRHKKLHTKREGPDSD

S. judaei PKKKPL-DGEYFTLKIRGRERFEMFRELHEALELKDTQHEKDSGESRAHSSYLKSKKGGSTSRHKKLHIKREGPDSD

H.glaber PKKKPL-DGEYFTLKIRGRERFEMFRELHEALELKDHQTEKEPGESRPHSSYLKSKKGQSTSCHKKLHFKKEGPDSD

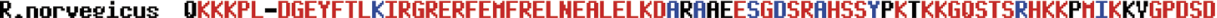

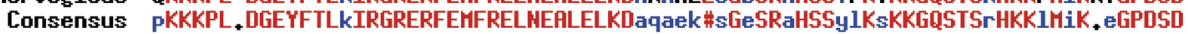

Figure 2. Amino acid sequence alignment of p53 protein with Homo sapiens, Myospalax baileyi, Eospalax cansus, Spalax judaei, Heterocephalus glaber and Rattus norvegicus. Amino acids with more than $90 \%$ identity are shown in red, 50\%-90\% identity in blue and the others in black. Arrows indicate that Myospalax baileyi has a mutation at residue 309 in the black box.

Gly292Glu, Pro297Leu, and Thr298Pro; and compared with $R$. norvegicus p53, the DNA-binding domain of $M$. baileyi has fifteen mutation sites: His108Arg, Gln113His, Ile126Pro, Ser127Cys, Thr146Asp, Trp174Cys, Gly183Ser, Pro199Leu, Tyr200Arg, Arg207Lys, Gln208His, Tyr227Cys, Lys233Asn, Asp266Asn, and Glu291Gly.

Multiple sequence alignment analysis showed that the mutation of codon 309 from glycine (G) to aspartic acid (D) in M. baileyi is unique to the plateau zokor compared to E. cansus, S. judaei and H. glaber p53. 
Table 1.

Likelihood values, parameter estimates, and sites under positive selection for $p 53$ in plateau zokor.

\begin{tabular}{|c|c|c|c|c|}
\hline Model & Parameter estimates & $\ln L$ & $\begin{array}{c}2 \Delta \ln L \\
(P \text {-value })\end{array}$ & Positive selection site \\
\hline Null A & $\begin{array}{l}p=0.785, p 1=0.215 \\
(p 2+p 3=0), \omega 0=0 \\
\omega 1=1.0, \omega 2=1.0\end{array}$ & -8750.89 & $0(P=1.000)$ & Not available \\
\hline Model A & $\begin{array}{l}p=0.785, p 1=0.215 \\
(p 2+p 3=0), \omega 0=0 \\
\omega 1=1.0, \omega 2=1.0\end{array}$ & -8750.89 & & Not found \\
\hline
\end{tabular}

\section{Positive selection sites analysis}

To detect the positively selected sites of $p 53$ in plateau zokor, we treated the plateau zokor branch as the foreground branch. Using the LRT based on the branch-site model for gene $p 53$, we observed that there are no positive selection sites in plateau zokor $p 53$ (table 1).

\section{Convergent evolution analysis}

To detect the sites that are convergent in subterranean rodents in response to hypoxic environments, we attempted to identify convergent changes by comparing ancestral and extant $p 53$ protein sequences. We observed that two sites experienced convergent evolution in subterranean rodents. The 78th position of $p 53$ was proline (P) on the ancestral branch. However, it was replaced by serine (S) on the subterranean rodent branch of $M$. baileyi and $S$. judaei. This change was caused by a $\mathrm{C}$ to $\mathrm{T}$ transition at the first position of the codon at site 78. In addition, the 84th position was alanine (A) on the ancestral branch, and it was replaced by proline $(\mathrm{P})$ in the subterranean rodent branch (fig. 3). According to a statistical test, the 78th and 84th positions were likely convergent sites, rather than chance substitution $(P<0.01)$.

\section{Expression of plateau zokor p53 under hypoxia}

As shown in fig. 4, the relative expression level of p53 mRNA in plateau zokor tissues was significantly higher in the high-altitude group $(3300 \mathrm{~m})$ than the lowaltitude group (2260 m) (liver: $t=7.50, P<0.0001$; lung: $t=17.41, P<0.0001$; stomach: $t=10.01, P<0.0001$; intestine: $t=12.95, P<0.0001$; skeletal muscle: $t=7.98, P<0.0001)$. However, there was no significant difference between the high- and low-altitude groups in rat (liver: $t=1.67, P=0.1131$; lung: $t=1.59$, $P=0.1283$; stomach: $t=0.75, P=0.4615$; intestine: $t=1.17, P=0.2577$; skeletal muscle: $t=0.53, P=0.5993)$. 


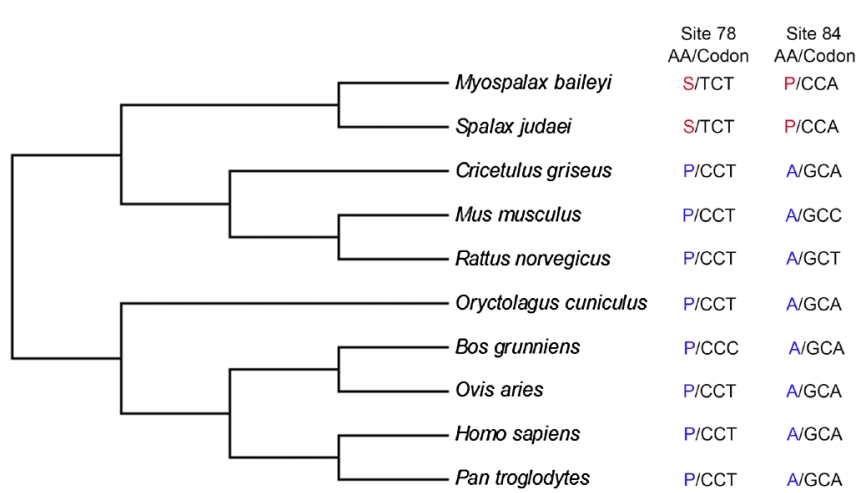

Figure 3. Evolution of convergent sites in $p 53$ sequences. Amino acids and codons of sites 78 and 84 are shown. Amino acids in Myospalax baileyi and Spalax judaei are highlighted in red, and in the other species in blue.

\section{Multiple alignment of HIF-1 $\alpha$ sequences}

The complete coding sequence (CDS) of $H$. sapiens HIF- $1 \alpha$ was $2481 \mathrm{bp}$, encoding 826 amino acids, that of $M$. baileyi was $2460 \mathrm{bp}$, encoding 819 amino acids, and of $R$. norvegicus it was 2472 bp, encoding 823 amino acids. The ODD domain consisted of 203, 201 and 202 amino acids in H. sapiens, M. baileyi and $R$. norvegicus, respectively.

Alignment by ClustalW2 found that in human, the motif at amino acids 397 to 436 is up to $90.00 \%$ identical with plateau zokor and rat, and the other motif in human (positions 507 to 579) is $97.26 \%$ and $95.89 \%$ identical with those in plateau zokor and rat, respectively. Compared with human, plateau zokor and rat had four common mutation sites within the motif 397 to 436: Asn416Asp, Asp421Glu, Glu427Asp, Leu436Phe, and they had two mutation sites in common within the motif 507 to 579: Tyr522Asp, Glu530Val. In addition, rat had a mutation of codon 552 from Thr (T) to Ala (A).

The sequence alignment showed that the amino acid sequences of the plateau zokor $H I F-1 \alpha$ ODD domain were $92.61 \%$ identical to those in rat. Compared with plateau zokor, rat had 12 mutation sites within the ODD domain: Asp440Asn, Asp441Glu, Thr444Asn, Asn465Ser, Pro480Ser, Asn481Ser, Ala482Pro, Glu486Gly, Thr552Ala, Ser586Asn, Asn589Ser, Ala599Val. The motif 397 to 436 of plateau zokor is $100 \%$ identical with that of rat, and the other motif (507 to 579) of plateau zokor is $98.63 \%$ identical with that of rat. Compared with plateau zokor, rat had one mutation sites within the motif 507 to 579: Thr552Ala (fig. 5).

\section{Discussion}

The plateau zokor is a specialized rat on the Qinghai-Tibet Plateau. This species was reported to have adaptations in a series of genes involved in hypoxia response, including p53 (Fan \& Shi, 1982; Zheng et al., 2011; Wang et al., 2012; Zhao et al., 

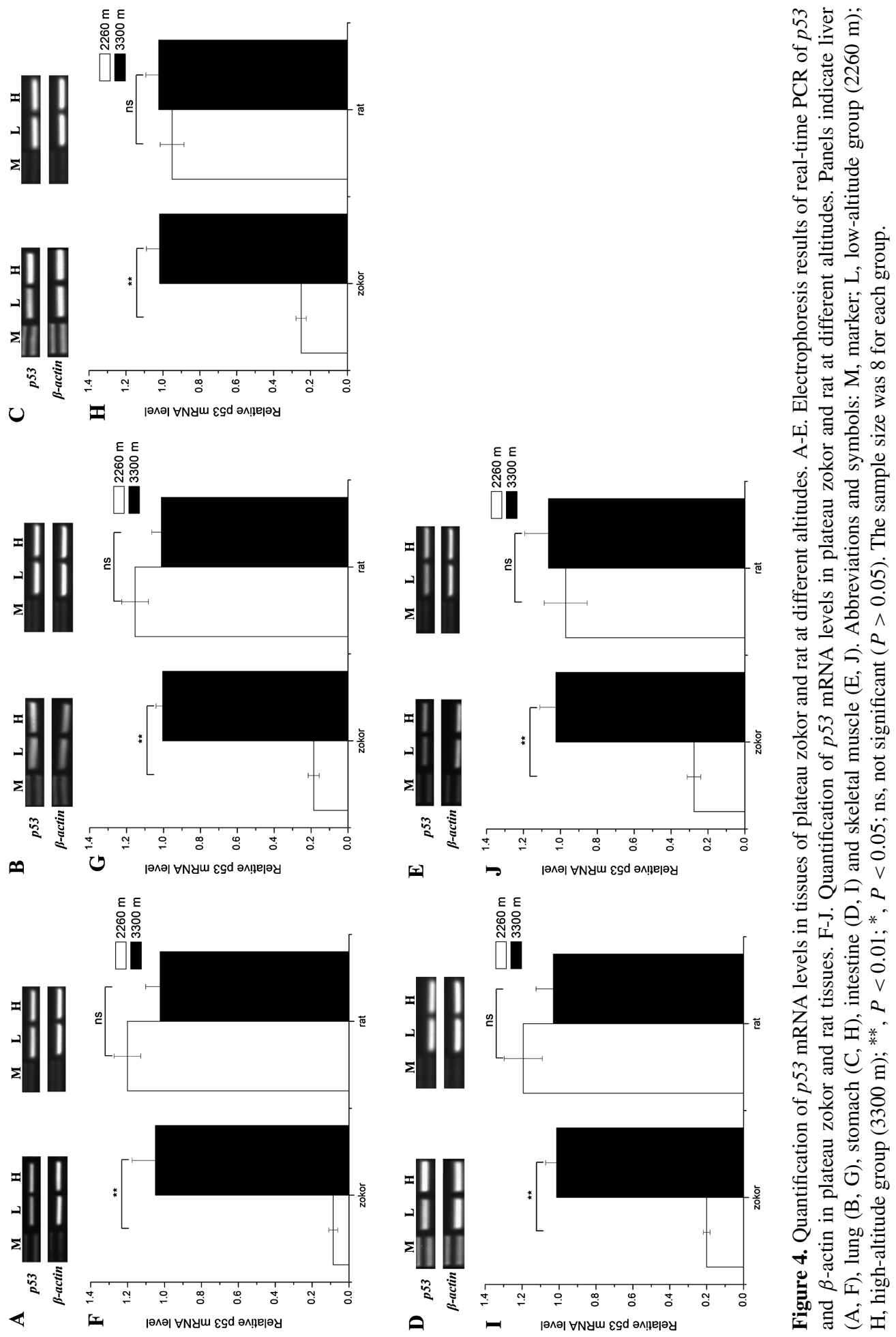


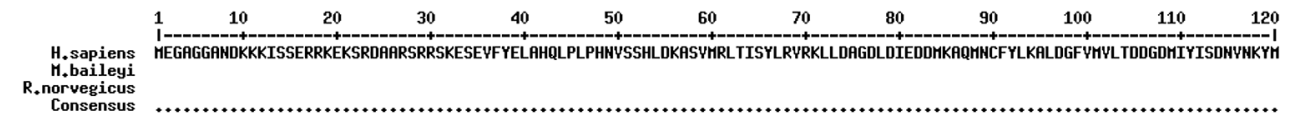

$\begin{array}{llllllllllll}121 & 130 & 140 & 150 & 160 & 170 & 180 & 190 & 200 & 210 & 220 & 230\end{array}$

H.sapiens GLTQFELTGHSYFDFTHPCDHEEMREMLIHRNGLYKKGKEQNTGRSFFLRHKCTLTSRGRTMNIKSATHKYLHCTGHIHUYDTHSNQPQCGYKKPPHTCLYLICEPIPHPSNIEIPLDSK H.baileyi

norvegicus

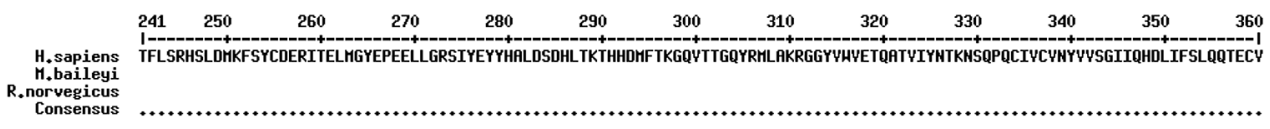

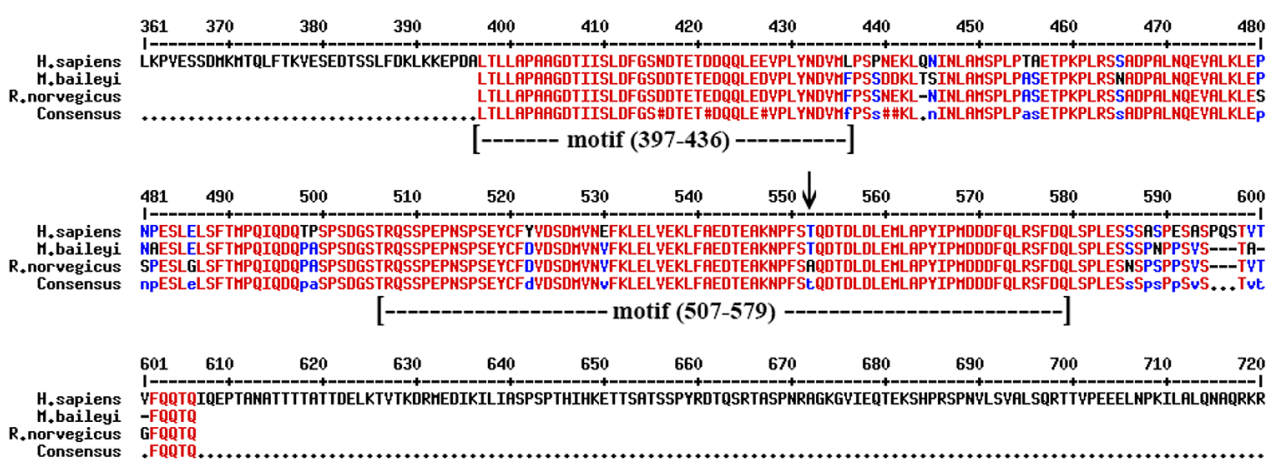

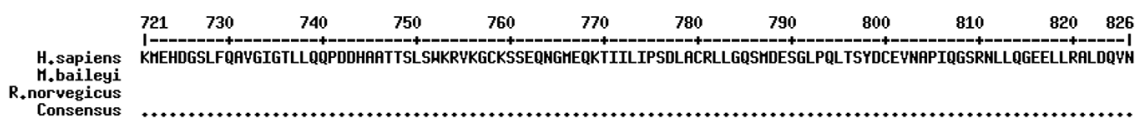

Figure 5. Amino acid sequence alignment of HIF-1 $\alpha$ ODD domain in Homo sapiens, Myospalax baileyi and Rattus norvegicus. Amino acids with more than $90 \%$ identity are highlighted in red, 50-90\% identity in blue and the others in black. The arrow indicates residue 552.

2013). According to our phylogenetic gene tree, the plateau zokor is placed with rodentia and shares many homologies with $E$. cansus. These two species are grouped on a small branch with $S$. judaei as sister group. In an analysis of reporter assays, $E$. cansus and $S$. judaei were grouped on a small branch subtended by plateau zokor (Zhao et al., 2013). These species all grouped into a large branch with $R$. norvegicus. Heterocephalus glaber belongs to the family of subterranean rodents; however, this species was far removed from other subterranean rodents. Studies of the phylogenetic relationship based on the genome and transcriptome analysis found that the plateau zokor diverged from the rat approximately 52 million years ago (Shao et al., 2015). A molecular phylogenetic tree based on the $p 53$ and cytochrome b protein sequences reported previously showed that plateau zokor and E. cansus were grouped on a branch with $S$. judaei as sister group (Zhao et al., 2013). Eospalax cansus is a typical subterranean rodent, living on the Loess Plateau in China (Li, 1989). Severe hypoxia and hypercapnia are common characteristics of burrows (Fan \& Shi, 1982; 
Nevo, 1999). During long-term evolution, M. baileyi and E. cansus developed a series of strategies to evolve adaptively and convergently in the hypoxic environment. They have a higher oxygen utilization rate in their tissues to adapt to hypoxic conditions, with a high oxygen pressure and saturation in arterial blood, and a low oxygen pressure and saturation in venous blood; the number of red blood cells, and the hemoglobin and myoglobin concentrations are also significantly higher. In addition, they have substitutions of amino acids in hemoglobin and myoglobin to increase the affinity to oxygen, and have enhanced their capacity to adapt to hypoxic environments (Wei et al., 2006a, b; Yang et al., 2006; Wang et al., 2008b). Thus, our results are consistent with previously published reports and imply that the molecular evolution of the $p 53$ gene in plateau zokor is similar to that in E. cansus, and this also indicates that subterranean rodents have evolved adaptively and convergently to hypoxia.

Acting as a tumour suppressor gene, $p 53$ has mutations that are also observed in approximately $50 \%$ of human cancers (Hernandez et al., 2003; Klein, 2004). Several mutations were reported at the mutation 'hotspots' at residues R175, G245, R248, R249, R273, and R282. The mutation occurs in more than $90 \%$ of human cancers (Bullock \& Fersht, 2001; Freed-Pastor \& Prives, 2012). Compared to the amino acid sequence of $p 53$ protein in E. cansus, S. judaei and H. glaber, we found that in plateau zokor codon 309 is mutated from glycine $(G)$ to aspartate (D); this mutation is unique for plateau zokor. We predict that the Gly309Asp mutation may play a significant role in the adaptation of the plateau zokor to its extreme environment, but this warrants further investigation. We also observed that the subterranean rodents (M. baileyi, E. cansus, S. judaei, H. glaber) had seven mutation sites in common compared to human $p 53$. There were two mutation sites in common with the human hotspots: Gln246Arg (248 in human) and His271 Arg (273 in human). These two mutations are reported to be the most frequently altered residues in $p 53$ protein. In $p 53$, these mutations cause a loss of wild-type $p 53$ tumour suppressor activity and promote tumourigenesis, resulting in cell cycle G1 arrest in cells (Cho et al., 1994; Hollstein et al., 1994; Vousden \& Lu, 2002; Menendez et al., 2006). Using site-directed mutagenesis to study the function of $p 53$, the Arg174Lys amino acid substitution in Spalax was found to reduce transcription of the apoptosis genes and enhance cell cycle arrest and $p 53$ stabilization/homeostasis genes (Ashur-Fabian et al., 2004; Avivi et al., 2007). The Ser104Asp amino acid substitution in plateau zokor activated the apoptosis genes and had no significant influence on cell cycle arrest genes, including $p 21$ and Mdm2 (Zhao et al., 2013). The mutations in different subterranean rodents were species-specific and could be attributed to their specific microenvironment. Therefore, we propose that the variations do not promote tumourigenesis in subterranean rodents, but enhance their adaptability to the hypoxic environment.

Subterranean rodents live in dark, hypoxic and hypercapnic underground environments (Fan \& Shi, 1982; Nevo, 1999, 2011; Nevo et al., 2001; Shams et al., $2005)$, and p53 plays an important role in hypoxic environments. Genome and 
transcriptome data have shown that $p 53$ is not positively selected in subterranean rodents (Kim et al., 2011; Fang et al., 2014), and previous studies have shown that plateau zokor $p 53$ does not have positively selected sites (Zhao et al., 2013). In this study, the LRT based on branch-site model A for gene $p 53$ indicated there are no positive selection sites in plateau zokor. In addition, we observed that $p 53$ amino acid sequences have two convergent sites in M. baileyi and $S$. judaei. We propose that the two substitution sites might enhance subterranean rodent adaptation to hypoxic environments.

Studies have demonstrated that the expression level of $p 53$ is related to the degree of hypoxia. Under normal conditions, wild-type $p 53$ is expressed at low levels in most cells and has a short half-life. In contrast, its level increases and stabilizes in response to hypoxia (Giaccia \& Kastan, 1998; Koumenis et al., 2001). However, later study showed that hypoxic induction of $p 53$ requires the concomitant induction of HIF-1 $\alpha$, demonstrating a direct interaction between $p 53$ and $H I F-1 \alpha$ (Hansson et al., 2002; Fels \& Koumenis, 2005; Sánchez-Puig et al., 2005). The oxygen-dependent degradation (ODD) domain lies within amino acids 401 to 603 in human HIF-1 $\alpha$ (Huang et al., 1998). In the ODD domain, the two proline residues (P402 and P564), play a pivotal role in regulating HIF-1 activity and expression by the ubiquitin-proteasome pathway, and act as switches for the oxygen-dependent regulation of HIF-1 $\alpha$ (Huang et al., 1998; Masson et al., 2001). Studies have iden-

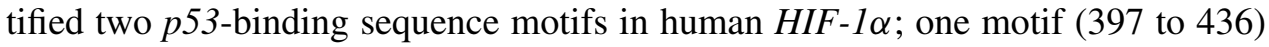
is adjacent to the ODD domain, the other motif (507 to 579) is coincident with the ODD domain of HIF-1 $\alpha$ (Hansson et al., 2002). Under normoxic conditions, the expression levels of $p 53$ and $H I F-1 \alpha$ are low. When the oxygen concentration decreases, $H I F-1 \alpha$ stabilizes, and the $p 53$ level remains low or lower than before. Under severe hypoxia or anoxic conditions, $p 53$ is accumulated and stabilizes and binds to the ODD domain of HIF-1 $\alpha$ (Fels \& Koumenis, 2005; Sánchez-Puig et al., 2005). When comparing oxygen levels at a mimicked altitude of $7000 \mathrm{~m}(8.0 \%$ $\mathrm{O}_{2}$ ) for $8 \mathrm{~h}$ with an altitude of $3352 \mathrm{~m}$, the $p 53$ expression level was significantly decreased in plateau zokor liver and significantly increased in rat. However, there was no significant difference between $10.8 \% \mathrm{O}_{2}$ and $16.0 \% \mathrm{O}_{2}$ (Wang et al., 2013; Zhao et al., 2013). In this study, we found that the relative expression levels of $p 53$ in the tissues of liver, lung, stomach, intestine and skeletal muscle of the plateau zokor are increased significantly from $2260 \mathrm{~m}$ to $3300 \mathrm{~m}$, but there was no significant difference under the same conditions in rat. Thus, the expression level of p53 showed different patterns among plateau zokor and rat in various tissues. This could be due to different regulatory mechanisms in the two species that are adaptive for the hypoxic environment. Doing a multiple sequence alignment of the two $p 53$ binding sequence motifs in $H I F-1 \alpha$, our results show that plateau zokor and rat are highly homologous to human, with the two proline residues (P402 and P564) not mutated in plateau zokor or rat. Compared with plateau zokor, rat had one mutation site within the motif 507 to 579: a polar amino acid (Thr, T) was substituted by a non-polar amino acid (Ala, A) in residue 552 in rat. The results imply that the 
substitution in the motif 507 to 579 decreases the affinity of $H I F-1 \alpha$ to $p 53$-binding sites in rat, and the $p 53$-binding sites in $H I F-1 \alpha$ of plateau zokor are stronger than that of rat.

\section{Acknowledgements}

This work was supported by the Natural Science Foundation of Qinghai Province (grant no. 2016-ZJ-901).

\section{References}

Arieli, R. \& Ar, A. (1981) Heart rate responses of the mole rat (Spalax ehrenbergi) in hypercapnic, hypoxic, and cold conditions. Physiol. Zool., 54, 14-21.

Arieli, R., Heth, G., Nevo, E. \& Hoch, D. (1986) Hematocrit and hemoglobin concentration in four chromosomal species and some isolated populations of actively speciating subterranean mole rats in Israel. Experientia, 42, 441-443.

Ashur-Fabian, O., Avivi, A., Trakhtenbrot, L., Adamsky, K., Cohen, M., Kajakaro, G., Joel, A., Amariglio, N., Nevo, E. \& Rechavi, G. (2004) Evolution of $p 53$ in hypoxia-stressed Spalax mimics human tumor mutation. Proc. Natl Acad. Sci. USA, 101, 12236-12241.

Avivi, A., Ashur-Fabian, O., Joel, A., Trakhtenbrot, L., Adamsky, K., Goldstein, I., Amariglio, N., Rechavi, G. \& Nevo, E. (2007) p53 in blind subterranean mole rats - loss-of-function versus gainof-function activities on newly cloned Spalax target genes. Oncogene, 26, 2507-2512.

Bargonetti, J., Manfredi, J.J., Chen, X., Marshak, D.R. \& Prives, C. (1993) A proteolytic fragment from the central region of $p 53$ has marked sequence-specific DNA-binding activity when generated from wild-type but not from oncogenic mutant $p 53$ protein. Gene Dev., 7, 2565-2574.

Ben-Shlomo, R. \& Maeda, N. (1989) Haptoglobin DNA polymorphism in subterranean mole rats of the Spalax ehrenbergi superspecies in Israel. Heredity, 62, 85-90.

Buffenstein, R. (2008) Negligible senescence in the longest living rodent, the naked mole-rat: insights from a successfully aging species. J. Comp. Physiol. B, 178, 439-445.

Buffenstein, R. \& Jarvis, J.U.M. (2002) The naked mole rat - a new record for the oldest living rodent. Sci. Aging Knowl. Environ., 2002, pe7. DOI:10.1126/sageke.2002.21.pe7.

Bullock, A.N. \& Fersht, A.R. (2001) Rescuing the function of mutant p53. Nat. Rev. Cancer, 1, 68-76.

Cho, Y., Gorina, S., Jeffrey, P.D. \& Pavletich, N.P. (1994) Crystal structure of a $p 53$ tumor suppressorDNA complex: understanding tumorigenic mutations. Science, 265, 346-355.

Corpet, F. (1988) Multiple sequence alignment with hierarchical clustering. Nucleic Acids Res., 16, 10881-10890.

Delaney, M.A., Nagy, L., Kinsel, M.J. \& Treuting, P.M. (2013) Spontaneous histologic lesions of the adult naked mole rat (Heterocephalus glaber): a retrospective survey of lesions in a zoo population. Vet. Pathol., 50, 607-621.

Edoute, Y., Arieli, R. \& Nevo, E. (1988) Evidence for improved myocardial oxygen delivery and function during hypoxia in the mole rat. J. Comp. Physiol. B, 158, 575-582.

Edrey, Y.H., Casper, D., Huchon, D., Mele, J., Gelfond, J.A., Kristan, D.M., Nevo, E. \& Buffenstein, R. (2012) Sustained high levels of neuregulin-1 in the longest-lived rodents; a key determinant of rodent longevity. Aging Cell, 11, 213-222.

El-Deiry, W.S. (1998) Regulation of p53 downstream genes. Semin. Cancer Biol., 8, 345-357.

Fan, N.C. \& Shi, Y.Z. (1982) A revision of the zokors of subgenus Eospalax. Acta Theriol. Sin., 2, 183-199. 
Fang, X., Nevo, E., Han, L., Levanon, E.Y., Zhao, J., Avivi, A., Larkin, D., Jiang, X., Feranchuk, S., Zhu, Y., Fishman, A., Feng, Y., Sher, N., Xiong, Z., Hankeln, T., Huang, Z., Gorbunova, V., Zhang, L., Zhao, W., Wildman, D.E., Xiong, Y., Gudkov, A., Zheng, Q., Rechavi, G., Liu, S., Bazak, L., Chen, J., Knisbacher, B.A., Lu, Y., Shams, I., Gajda, K., Farre, M., Kim, J., Lewin, H.A., Ma, J., Band, M., Bicker, A., Kranz, A., Mattheus, T., Schmidt, H., Seluanov, A., Azpurua, J., McGowen, M.R., Jacob, E.B., Li, K., Peng, S., Zhu, X., Liao, X., Li, S., Krogh, A., Zhou, X., Brodsky, L. \& Wang, J. (2014) Genome-wide adaptive complexes to underground stresses in blind mole rats Spalax. Nat. Commun., 5, 3966. DOI:10.1038/ncomms4966.

Fels, D.R. \& Koumenis, C. (2005) HIF-1 $\alpha$ and i: the ODD couple? Trends Biochem. Sci., 30, 426-429.

Freed-Pastor, W.A. \& Prives, C. (2012) Mutant p53: one name, many proteins. Gene Dev., 26, 12681286.

Giaccia, A.J. \& Kastan, M.B. (1998) The complexity of p53 modulation: emerging patterns from divergent signals. Gene Dev., 12, 2973-2983.

Graeber, T.G., Osmanian, C., Jacks, T. \& Housman, D.E. (1996) Hypoxia-mediated selection of cells with diminished apoptotic potential in solid tumours. Nature, 379, 88-91.

Gurnett, A.M., O'Connell, J.P., Harris, D.E., Lehmann, H., Joysey, K.A. \& Nevo, E. (1984) The myoglobin of rodents: Lagostomus maximus (viscacha) and Spalax ehrenbergi (mole rat). J. Protein Chem., 3, 445-454.

Hansson, L.O., Friedler, A., Freund, S., Rüdiger, S. \& Fersht, A.R. (2002) Two sequence motifs from $H I F-1 \alpha$ bind to the DNA-binding site of p53. Proc. Natl Acad. Sci. USA, 99, 10305-10309.

Hernandez, I., Maddison, L.A., Wei, Y., DeMayo, F., Petras, T., Li, B., Gingrich, J.R., Rosen, J.M. \& Greenberg, N.M. (2003) Prostate-specific expression of p53 R172L differentially regulates $p 21$, Bax, and $m d m 2$ to inhibit prostate cancer progression and prolong survival. Mol. Cancer Res., 1, 1036-1047.

Hollstein, M., Rice, K., Greenblatt, M.S., Soussi, T., Fuchs, R., Sørlie, T., Hovig, T., Smith-Sorensen, B., Montesano, R. \& Harris, C.C. (1994) Database of $p 53$ gene somatic mutations in human tumors and cell lines. Nucleic Acids Res., 22, 3551-3555.

Huang, L.E., Gu, J., Schau, M. \& Bunn, H.F. (1998) Regulation of hypoxia-inducible factor $1 \alpha$ is mediated by an $\mathrm{O}_{2}$-dependent degradation domain via the ubiquitin-proteasome pathway. Proc. Natl Acad. Sci. USA, 95, 7987-7992.

Jeanmougin, F., Thompson, J.D., Gouy, M., Higgins, D.G. \& Gibson, T.J. (1998) Multiple sequence alignment with Clustal X. Trends Biochem. Sci., 23, 403-405.

Ke, Q. \& Costa, M. (2006) Hypoxia-inducible factor-1 (HIF-1). Mol. Pharmacol., 70, 1469-1480.

Kim, E.B., Fang, X., Fushan, A.A., Huang, Z., Lobanov, A.V., Han, L., Marino, S.M., Sun, X., Turanov, A.A., Yang, P., Yim, S.H., Zhao, X., Kasaikina, M.V., Stoletzki, N., Peng, C., Polak, P., Xiong, Z., Kiezun, A., Zhu, Y., Chen, Y., Kryukov, G.V., Zhang, Q., Peshkin, L., Yang, L., Bronson, R.T., Buffenstein, R., Wang, B., Han, C., Li, Q., Chen, L., Zhao, W., Sunyaev, S.R., Park, T.J., Zhang, G., Wang, J. \& Gladyshev, V.N. (2011) Genome sequencing reveals insights into physiology and longevity of the naked mole rat. Nature, 479, 223-227.

Kinzler, K.W. \& Vogelstein, B. (1996) Lessons from hereditary colorectal cancer. Cell, 87, 159-170.

Klein, G. (2004) Cancer, apoptosis, and nonimmune surveillance. Cell Death Differ, 11, 13-17.

Kleinschmidt, T., Nevo, E. \& Braunitzer, G. (1984) The primary structure of the hemoglobin of the mole rat (Spalax ehrenbergi, Rodentia, chromosome species 60). Hoppe Seyler Z. Physiol. Chem., 365, 531-538.

Kleinschmidt, T., Nevo, E., Goodman, M. \& Braunitzer, G. (1985) Mole rat hemoglobin: primary structure and evolutionary aspects in a second karyotype of Spalax ehrenbergi, Rodentia, $(2 n=$ 52). Biol. Chem. Hoppe Seyler, 366, 679-686. 
Koumenis, C., Alarcon, R., Hammond, E., Sutphin, P., Hoffman, W., Murphy, M., Derr, J., Taya, Y., Lowe, S.W., Kastan, M. \& Giaccia, A. (2001) Regulation of p53 by hypoxia: dissociation of transcriptional repression and apoptosis from p53-dependent transactivation. Mol. Cell. Biol., 21, 1297-1310.

Kumar, S., Stecher, G. \& Tamura, K. (2016) MEGA7: molecular evolutionary genetics analysis version 7.0 for bigger datasets. Mol. Biol. Evol., 33, 1870-1874.

Li, D.H. (1989) Economic Animal Fauna of Qinghai. Qinghai People's Publishing House, Xining, China.

Livak, K.J. \& Schmittgen, T.D. (2001) Analysis of relative gene expression data using real-time quantitative PCR and the $2^{-\triangle \Delta C T}$ method. Methods, 25, 402-408.

Masson, N., Willam, C., Maxwell, P.H., Pugh, C.W. \& Ratcliffe, P.J. (2001) Independent function of two destruction domains in hypoxia-inducible factor- $\alpha$ chains activated by prolyl hydroxylation. EMBO J., 20, 5197-5206.

Menendez, D., Inga, A. \& Resnick, M.A. (2006) The biological impact of the human master regulator p53 can be altered by mutations that change the spectrum and expression of its target genes. Mol. Cell. Biol., 26, 2297-2308.

Nevo, E. (1999) Mosaic Evolution of Subterranean Mammals: Regression, Progression, and Global Convergence. Oxford University Press, Oxford, UK.

Nevo, E. (2011) Evolution under environmental stress at macro- and microscales. Genome Biol. Evol., 3, 1039-1052.

Nevo, E., Ivanitskaia, E. \& Beiles, A. (2001) Adaptive Radiation of Blind Subterranean Mole Rats: Naming and Revisiting the Four Sibling Species of the Spalax ehrenbergi Superspecies in Israel: Spalax galili $(2 n=52)$, S. golani $(2 n=5)$, S. carmeli $(2 n=58)$ and S. judaei $(2 n=60)$. Backhuys Publishers, Leiden, Netherlands.

Pavletich, N.P., Chambers, K.A. \& Pabo, C.O. (1993) The DNA-binding domain of p53 contains the four conserved regions and the major mutation hot spots. Gene Dev., 7, 2556-2564.

Pevet, P., Heth, G., Hiam, A. \& Nevo, E. (1984) Photoperiod perception in the blind mole rat (Spalax ehrenbergi, Nehring): involvement of the Harderian gland, atrophied eyes, and melatonin. J. Exp. Zool., 232, 41-50.

Resnick, M.A. \& Inga, A. (2003) Functional mutants of the sequence-specific transcription factor $p 53$ and implications for master genes of diversity. Proc. Natl Acad. Sci. USA, 100, 9934-9939.

Resnick, M.A., Tomso, D., Inga, A., Menendez, D. \& Bell, D. (2005) Functional diversity in the gene network controlled by the master regulator $p 53$ in humans. Cell Cycle, 4, 1026-1029.

Saitou, N. \& Nei, M. (1987) The neighbor-joining method: a new method for reconstructing phylogenetic trees. Mol. Biol. Evol., 4, 406-425.

Sánchez-Puig, N., Veprintsev, D.B. \& Fersht, A.R. (2005) Binding of natively unfolded HIF-1 $\alpha$ ODD domain to p53. Mol. Cell., 17, 11-21.

Shams, I., Avivi, A. \& Nevo, E. (2004) Hypoxic stress tolerance of the blind subterranean mole rat: expression of erythropoietin and hypoxia-inducible factor $1 \alpha$. Proc. Natl Acad. Sci. USA, 101, 9698-9703.

Shams, I., Nevo, E. \& Avivi, A. (2005) Erythropoietin receptor spliced forms differentially expressed in blind subterranean mole rats. FASEB J., 19, 1749-1751.

Shao, Y., Li, J.X., Ge, R.L., Zhong, L., Irwin, D.M., Murphy, R.W. \& Zhang, Y.P. (2015) Genetic adaptations of the plateau zokor in high-elevation burrows. Sci. Rep., 5, 17262. DOI:10.1038/ srep17262. 
Tamura, K., Peterson, D., Peterson, N., Stecher, G., Nei, M. \& Kumar, S. (2011) MEGA5: molecular evolutionary genetics analysis using maximum likelihood, evolutionary distance, and maximum parsimony methods. Mol. Biol. Evol., 28, 2731-2739.

Thut, C.J., Chen, J.L., Klemm, R. \& Tijan, R. (1995) p53 transcriptional activation mediated by coactivators TAF (II) 40 and TAF (II) 60. Science, 267, 100-104.

Tian, X., Azpurua, J., Hine, C., Vaidya, A., Myakishev-Rempel, M., Ablaeva, J., Mao, Z.Y., Nevo, E., Gorbunova, V. \& Seluanov, A. (2013) High-molecular-mass hyaluronan mediates the cancer resistance of the naked mole rat. Nature, 499, 346-349.

Vousden, K.H. \& Lu, X. (2002) Live or let die: the cell's response to p53. Nat. Rev. Cancer, 2, 594604.

Walker, K.K. \& Levine, A.J. (1996) Identification of a novel p53 functional domain that is necessary for efficient growth suppression. Proc. Natl Acad. Sci. USA, 93, 15335-15340.

Wang, M.Y., Zhao, Y., Zhang, S.T., Chen, X.Q. \& Du, J.Z. (2013) The regulation of expression for p53 and its target genes in simulate hypoxia. China J. Appl. Physiol., 29, 136-138.

Wang, X.J., Wei, D.B., Wei, L., Qi, X.Z., Zhu, S.H. \& Rao, X.F. (2008a) Characteristics of pulmonary acinus structure in the plateau zokor (Myospalax baileyi) and plateau pika (Ochotona curzniae). Acta Zool. Sin., 54, 531-539.

Wang, X.J., Wei, D.B., Wei, L., Zhang, J.M. \& Yu, H.Y. (2008b) Physiological character of erythrocyte adapting to hypoxia in plateau zokor and plateau pika. Sichuan J. Zool., 27, 1100-1103.

Wang, Z.L., Chen, Y., Yang, J., Chen, W.J., Zhang, Y.M. \& Zhao, X.Q. (2012) cDNA cloning and expression of erythropoietin in the plateau zokor (Myospalax baileyi) from the Qinghai-Tibet Plateau. Chin. Sci. Bull., 57, 997-1006.

Wang, Z.W., Zeng, J.X. \& Han, Y.C. (1979) Studies on gas metabolism of pika (Ochotona) and plateau zokor (Myospalax baileyi). Acta Zool. Sin., 25, 75-84.

Wei, D.B. \& Ma, J.B. (2001a) Comparison of the content of myoglobin and lactate dehydrogenase in cardiac and skeleton muscle of plateau zorkor and mouse. J. Qinghai Univ., 19, 20-21.

Wei, D.B. \& Wei, L. (2001b) The mensuration results of the number of red cell and the content of hemoglobin and myoglobin in plateau zokor. J. Qinghai Univ., 19, 1-2.

Wei, D.B., Wei, L., Zhang, J.M. \& Yu, H.Y. (2006a) Blood-gas properties of plateau zokor (Myospalax baileyi). Comp. Biochem. Phys. A, 145, 372-375.

Wei, L., Yu, H.Y., Zhang, J.M., Mu, J.Y. \& Wei, D.B. (2006b) Seasonal changes of myoglobin in cardiac and skeleton muscle of Plateau Zokor (Myospalax baileyi). J. Qinghai Univ., 24, 34-36.

Widmer, H.R., Hoppeler, H., Nevo, E., Taylor, C.R. \& Weibel, E.R. (1997) Working underground: respiratory adaptations in the blind mole rat. Proc. Nat. Acad. Sci. USA, 94, 2062-2067.

Yang, J., Li, J.G., He, J.P. \& Zhang, Y.L. (2006) Blood composition and its relationship with hypoxia adaptation in Gansu Zokor. Chin. J. Zool., 41, 112-115.

Yang, Z.H. (2007) PAML 4: phylogenetic analysis by maximum likelihood. Mol. Biol. Evol., 24, 1586-1591.

Zhang, J. \& Kumar, S. (1997) Detection of convergent and parallel evolution at the amino acid sequence level. Mol. Biol. Evol., 14, 527-536.

Zhang, J.Z., Nielsen, R. \& Yang, Z.H. (2005) Evaluation of an improved branch-site likelihood method for detecting positive selection at the molecular level. Mol. Biol. Evol., 22, 2472-2479.

Zhao, K., Chai, X., Johnston, K., Clements, A. \& Marmorstein, R. (2001) Crystal structure of the mouse $p 53$ core DNA-binding domain at $2.7 \AA$ Å resolution. J. Biol. Chem., 276, 12120-12127.

Zhao, Y., Ren, J.L., Wang, M.Y., Zhang, S.T., Liu, Y., Li, M., Cao, Y.B., Zu, H.Y., Chen, X.Q., Wu, C.I., Nevo, E., Chen, X.Q. \& Du, J.Z. (2013) Codon 104 variation of p53 gene provides adaptive 
apoptotic responses to extreme environments in mammals of the Tibet plateau. Proc. Natl Acad. Sci. USA, 110, 20639-20644.

Zheng, Y.N., Zhu, R.J., Wang, D.W., Wei, L. \& Wei, D.B. (2011) Gene coding and mRNA expression of vascular endothelial growth factor as well as microvessel density in brain of plateau zokor: comparison with other rodents. Acta Physiol. Sin., 63, 155-163. 


\section{Table S1.}

Species examined in this study.

\begin{tabular}{ll}
\hline Species name & Accession Number \\
\hline Homo sapiens & DQ263704 \\
Rattus norvegicus & AH002222 \\
Mus musculus & AB020317 \\
Eospalax cansus & JX998172 \\
Spalax judaei & AJ783406 \\
Heterocephalus glaber & KM486789 \\
Cricetulus griseus & U50395 \\
Microtus oeconomus & JX998171 \\
Cavia porcellus & NM_001172740 \\
Meriones unguiculatus & AB033632 \\
Mesocricetus auratus & NM_001281661 \\
Rhesus monkey & L20442 \\
Macaca fascicularis & $\mathrm{U} 48957$ \\
Macaca mulatta & $\mathrm{U} 48956$ \\
Tupaia chinensis & NM_001287369 \\
Canis lupus familiaris & AB020761 \\
Felis catus & D26608 \\
Delphinapterus leucas & AF475081 \\
Sus scrofa & NM_213824 \\
Ovis aries & FJ855223 \\
Bubalus bubalis & JF792632 \\
Bos taurus & X81704 \\
Bos grunniens & KF682140 \\
Cynops orientalis & HM627863 \\
Xenopus laevis & X77546 \\
Oryzias latipes & U57306 \\
Platichthys flesus & Y08919 \\
Oncorhynchus mykiss & NM_001124692 \\
Pelteobagrus fulvidraco & HQ419002 \\
Ictalurus punctatus & NM_001200076 \\
Strongylocentrotus purpuratus & JF704085 \\
Brachionus koreanus & KJ526612 \\
Spodoptera frugiperda & HM773026 \\
Bombyx mori & HM773025 \\
Marsupenaeus japonicus & AB559569 \\
\hline & \\
\hline &
\end{tabular}

